\title{
Dimensioning and Assessment of Protected Converged Optical Access Networks
}

\author{
Arslan Shahid and Carmen Mas Machuca, Senior Member IEEE
}

\begin{abstract}
Optical access networks are continuously evolving towards next generation solutions offering much higher bandwidth per end point and considerably longer optical reach. Their dimensioning and planning should be accurate and as close to realistic values as possible to become useful to network operators. This work presents a Converged Access Network Planning and Dimensioning Tool for planning and dimensioning of networks for Fixed Mobile Convergence based on Geographical Information Systems (GIS). These networks connect to the Central Office (CO), end points requiring different capacity and reliability constraints. This tool proposes a new clustering algorithm to decrease fiber and duct length. Furthermore, five protection schemes have been proposed, modelled and compared in Dense Urban, Urban and Rural areas to improve connection availability to the more availability demanding end points. The proposed assessment methodology compares the following parameters: component cost, power consumption, connection availability, indirect improvement in connection availability of residential users, Failure Impact Factor (FIF) and protection fiber length required per Macro Base Station (MBS). The paper also includes a consolidated comparative analysis to find the best solution fulfilling specific requirements of any network/ service provider.
\end{abstract}

Index Terms-Next Generation Optical Access (NGOA), techno-economics, Converged access network, Protection schemes

\section{INTRODUCTION}

$\mathrm{O}$ ptical access networks can nowadays offer much longer reach and higher bandwidth communication than what it was few years ago. However, as the client count of access networks increases thanks to the increase of the delivered bandwidth, the impact of failure also increases. In this network centric society, the uninterrupted access to the network services is becoming vital and operators are now also considering to protect their access networks in addition to their aggregation and core networks. However, the cost factor is still very important due to the relatively low cost sharing of the access segment. One option to reduce cost is to use the same Optical Distribution Network (ODN) to interconnect fixed end points (e.g., Cabinets, buildings) as well as Macro Base Stations (MBS) at the so-called converged optical access networks.

A. Shahid was with the Chair of Communication Networks (LKN), Technische Universität München (TUM), Germany and is currently with Military College of Signals (MCS) a constituent campus of National University of Sciences and Technology (NUST), Pakistan.

Dr. C. Mas Machuca is with Chair of Communication Networks (LKN), Technische Universität München (TUM), Germany.
However, for this purpose, the network architecture should be able to offer different granularities in terms of bandwidth, connection reliability etc. Some new Next Generation Optical Access Network (NGOA) architectures [1] offer the expected longer reach and bandwidth levels. However, they are initially unprotected and hence in this paper we propose different protection mechanisms to increase their connection availability.

Planning and dimensioning of access networks should be as accurate and realistic as possible. This is either based on geometric models or geographic models.

- Geometric models like the Triangle model [2], the Simplified Street Length Model [3], Gabriel graphs [4], and TITAN [5] are easy to use but may lead to inaccurate results/ estimations especially for uneven distributed data, which is the case in most practical cases. Geometric models are using only the area-wide average parameters, and not their local characteristics. In practice, the areas where optical access networks are deployed are not evenly populated and the fiber trenching is constrained by various local conditions, e.g., parks, rivers, railways or highways. This is a reason why the geometric models cannot contribute to the accurate estimation of the deployment cost [6].

- Geographic models are the most preferred by operators, because of their high accuracy which ensures getting realistic results. These models can be used to select the right technology by evaluating all expenditures: Capital Expenditures (CAPEX), Implementation Expenditures (IMPEX) and Operational Expenditures (OPEX). The proposed methodology directly operates on available geospatial representation of the service area, which allows providing valid access network topologies. These topologies can be used as reliable and accurate base for trenching, fiber length and Remote Nodes (RNs) location planning. Geographical models also allow easier layout of network infrastructure and reduce IMPEX.

Some work has been presented on access planning using geographic models [7]. However, none of the existing dimensioning solutions address all the following aspects at once: (i) providing step by step dimensioning process description, (ii) completeness/ breakdown of information about shopping list (e.g., trenching diameter/ depth, tube sizes, etc.), (iii) ability to remove any inconsistent data from geographic database (e.g., like free standing features, dangles, cul-de-sacs), (iv) coping with two or more stage splitting of NGOA 
networks, (v) application to Fixed Mobile Convergence, (vi) improved clustering methodology considering street-aware distances instead of Euclidean distances reducing required fiber and duct and last but not least (vi) implementation of protection schemes.

The planning and assessment methodology proposed in this paper have been applied to different protection schemes aiming at offering protection to some end points, in this case, to the MBS (i.e. MBS have higher availability requirements than residential users). The assessment of the different protection schemes has been done in three different areas: Dense Urban, Urban and Rural areas.

It is important to mention that the proposed planning methodology is not limited to NGOAs, but it can be applied to other access networks using other technologies by defining the number of remote nodes, their splitting ratio, required bandwidth, etc.

The paper is organized as follows: Section II introduces the generic NGOA architecture. Section III describes the complete dimensioning/planning methodology. Section IV presents the different protection schemes. Section V introduces the assessment methodology and the considered criteria. Section VI presents the consolidated analysis of the proposed protection schemes. Finally Section VII concludes the paper.

\section{NGOA FOR CONVERGED ACCESS NETWORKS}

Converged Access Networks aim at connecting different types of endpoints (e.g., buildings and MBS) with the same ODN. Furthermore, node consolidation [8] is considered by operators in order to reduce the number of central offices and hence reduce OPEX, when using NGOA allowing longer reach and higher client count. Hence, the remaining $\mathrm{CO}$ (referred as Main Central Office (MCO)) are now able to serve much larger service areas and more customers, but at the cost of decreasing the connection availability due to the longer distances, and increasing the failure impact [9].

Several architectures have been proposed in literature: NGPON2 [1], WDM-PON[1], HPON [1],[10]. Most of these architectures have more than one splitting stage. Although all the architectures can be used by the proposed planning tool, this explanation will refer to HPON (depicted in Fig. 1) as it allows reusing existing ODN while fulfilling NGOA requirements. The Optical Line Terminal (OLT) is placed at the MCO. The architecture has two stages of remote nodes: The first stage denoted by "RN1" uses WDM filters (e.g., Arrayed Waveguide Gratings (AWGs)) for de-/multiplexing the downstream and upstream wavelengths. Compared to Power Splitters (PS), AWG has lower insertion loss and adds system integrity through wavelength separation. The second stage of remote nodes denoted by "RN2" are PSs. As opposed to MBS that have dedicated wavelength, users connected to the PS share the wavelength capacity by using Time Division Multiple Access (TDMA) scheme. This work considers MBS backhauled to the OLT and hence, one wavelength per MBS has been considered. However, the planning can be easily changed to more wavelengths per MBS for fronthaul solutions [14].

System guarantees provisioning wavelength capacity B (e.g.,
$10 \mathrm{Gbps}$ ) to each MBS or business user and $B / N$ to each residential user with $N$ being the splitting ratio of the PS.

The two stage architecture considers three fiber sections as shown in Fig. 1 (i) Feeder Fiber (FF) is the fiber from the MCO to AWG (ii) Distribution Fiber (DF) is the fiber from AWG to PS or MBS, and (iii) Last Mile Fiber (LMF) is the fiber from PS to the residential users ONU. This architecture has a tree topology where the OLT is at the root and is connected to AWGs, which are further connected to MBSs and PSs. This paper focuses to the scenario of having one MCO, hence distance to neighboring MCOs is very long due to node consolidation.

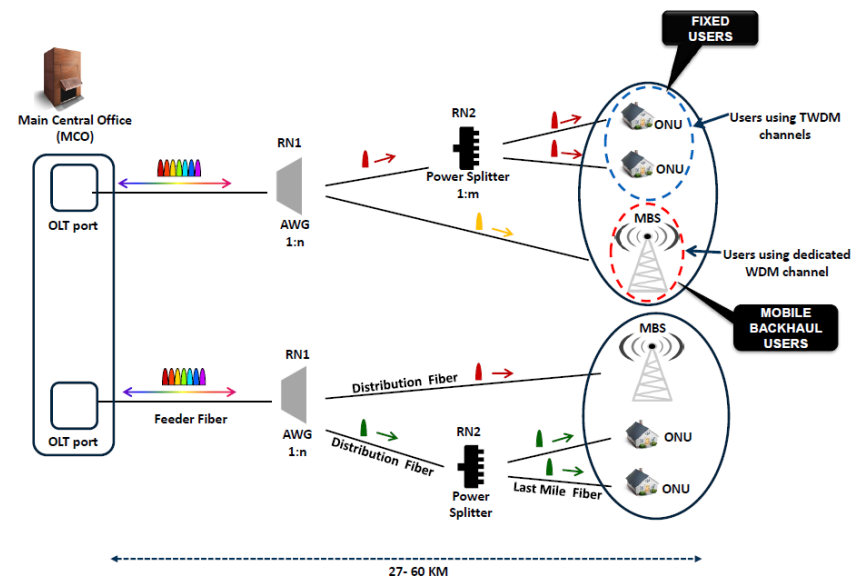

Figure 1 Two stage access HPON architecture: OLT (Optical Line Terminal); RN (Remote Node); AWG (Arrayed waveguide Grating); WDM (Wavelength Division Multiplexing); TDM (Time Division Multiplexing); ONU (Optical Network Unit)

\section{Dimensioning Methodology}

This section introduces step by step the methodology to dimension a converged access network (with or without protection) on a real area. The steps of adopted methodology are:

- $\quad$ Select the area of study from Open Street Map (osm), which is a free GIS database from www.openstreetmap.org.

- Extract buildings data and road/street data, in our case, using ArcGIS (arcgis.com) which allows working with osm maps [13].

- For every building select a node (e.g., center or closest to the street) and associate it to its ONU location since in this case Fiber To The Building/Home (FTTB/FTTH) has been considered (Fig. 2(a)).

- Unless the MBS locations are known (e.g., provided by the mobile operator), MBS can be placed based on Voronoi model, or as a regular fishnet distribution given the inter MBS distance (red stars in Fig. 2(b)). In this study, the MBS density is expected to influence more than the MBS distribution itself since the ratio of buildings vs. MBS is high. The MBS are then associated to the nearest street node as shown as green stars in Fig. 2(b).

- $\quad 1^{\text {st }}$ stage clustering: As shown in Figure 1, buildings are connected to PS. Hence, given the PS splitting ratio and the port usage, buildings are clustered (Fig. 2(c)). The 
proposed clustering algorithm has been designed to generate clusters of fixed size with the possibility to dynamically adjust individual cluster size and/or total number of clusters to maintain cluster quality and to reduce the required infrastructure. The proposed clustering is presented in the next Section. The centroid of each cluster is the best location for the PS. However, based on the experience from operators, they are relocated to nearest intersection node (street crossing) because it increases accessibility and facilitates finding alternative paths required for protection (shown as black triangles in Fig. 2(d)).

- $\quad 2^{\text {nd }}$ stage clustering: PS and MBS are clustered to AWGs based on the number of wavelengths and the port usage of the AWG (Fig. 2(e)). Although AWGs are initially placed at each cluster centroid, they are relocated to the nearest intersection node (yellow round shape).

- Compute the fiber layout of each fiber section (i.e. FF, DF and LMF). The fiber layout can be computed using different approaches: e.g., simple shortest path, shortest path with maximum duct sharing [11][12]. Based on the layout, the fiber required for each section as well as the duct can be computed. The duct is calculated by merging and dissolving all fiber paths. Furthermore, the cable size for each street segment can be computed as shown in Fig. 2(f).

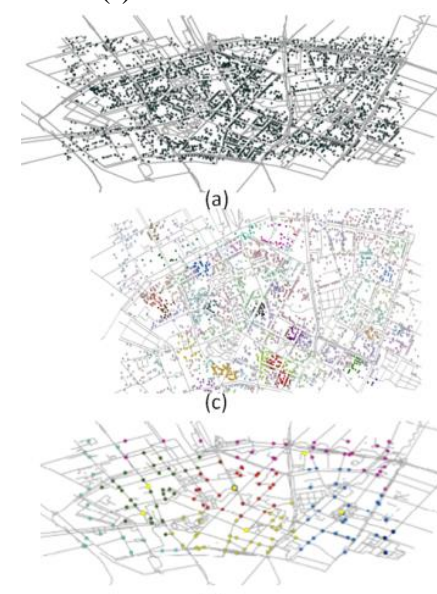

(e)

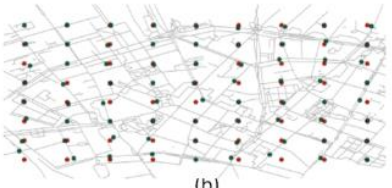

(b)

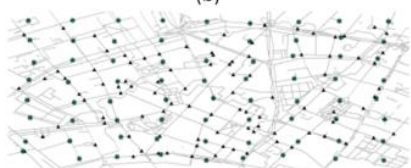

(d)

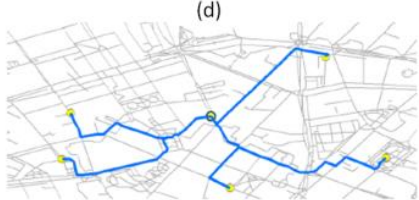

Figure 2 Planning methodology

As a result of the network planning, the number and location of all components (MCO, AWGs and PSs) as well as required fiber and cables in terms of different lengths and sizes are obtained. Although the fiber is aimed to be minimized, the maximum reach must not be exceeded. In case it is exceeded, reach extenders should be placed at the right remote nodes.

\section{PROPOSED CLUSTERING ALGORITHM}

Most of the clustering approaches considered in literature are based on K-means clustering, i.e. on Euclidean distances which may differ significantly from street-aware distances, especially in rural areas. Furthermore, K-means does not consider the impact that cluster sizes and distance have on infrastructure costs (e.g., adding a cluster, i.e. a splitter, may help to reduce costs, i.e. shorter distances).

In this section we propose a clustering algorithm that generates clusters given the splitting ratio with the possibility to dynamically adjust individual cluster size and/or total number of clusters to reduce the required infrastructure. It considers the following aspects:

- Cost Matrix (CM): It is the matrix which stores the street-aware distances from one Cluster Element (CE) to the rest of the CEs. CEs are the elements that should be clustered (e.g., buildings at 1st stage clustering).

- Initialization method: Ascending order: One critical decision for any clustering algorithm is to identify from which node or seed master to start for building clusters. The proposed algorithm has been designed to compare different approaches: (i) considering distance from the seed master to the farthest seed member (ii) considering the aggregate distance from the seed master to all its members. Both approaches consider either ascending or descending sorting order. We have identified that for the proposed DU, $\mathrm{U}$ or $\mathrm{R}$ areas with reasonable roads infrastructure, selecting aggregate distance with ascending order yields the best results [13].

- Penalty Matrix: It is the matrix that stores the costs for the defined initialization method by considering the resulting clusters and largest cluster size.

- Cost Threshold: This parameter defines the expected compactness of the cluster, and whenever it is exceeded, a new cluster is created instead of adding too dispersed elements. The impact of such threshold has been depicted in Fig. 3 (b) where savings on $28 \%$ on infrastructure cost can be achieved by adding $7 \%$ more clusters.

The proposed clustering algorithm is depicted in Fig. 3 (a). This algorithm has been compared to K-means and the impact on the number of clusters as depicted in Fig. 3 (b-c). In this case, PSs are clustered given the splitting ratio of AWGs. Fig. 3 (b) shows 6 different clusters and the length from each AWG to each PS of its cluster in meters. Each cluster may have different number of PSs (the more colors, the more PS are in the same cluster) and the PS have been ordered based on their distance to their AWG. It can be observed, that the last cluster contains less PS and have significant longer distance. Fig. 3 (c) shows the advantage of the proposed scheme which by increasing just by one the number of clusters; it reduces significantly the distance to the AWG. For that purpose, a comparison of the proposed algorithm using geographic distances (CL Street Aware) and using Euclidean distances ( $C L$ Euclidean) have been performed to the K-means for a DU area with $72 \mathrm{MBS}$ as depicted in Fig. 3 (d). It can be observed that the infrastructure cost required per MBS is reduced by $6 \%$ with respect to K-means and by $4.7 \%$ with respect to Euclidean distances. It is pertinent to highlight that different $\mathrm{K}$ values were used and the performance of each $\mathrm{K}$ value was evaluated. For fair comparison, the optimal/ best solution found by K-means was selected and compared with our clustering algorithm which yields better and consistent results and most importantly does not require any sub-distance optimization. 


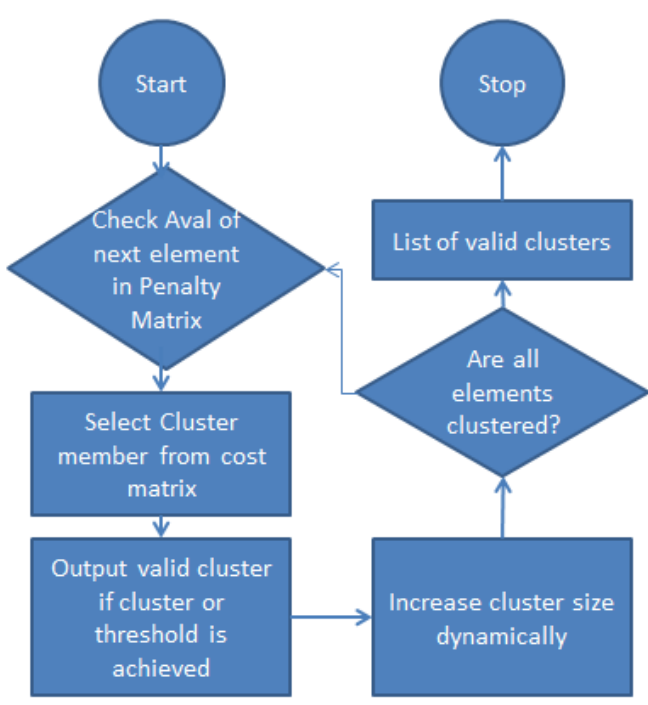

a) Proposed CMPMT algorithm

Meters

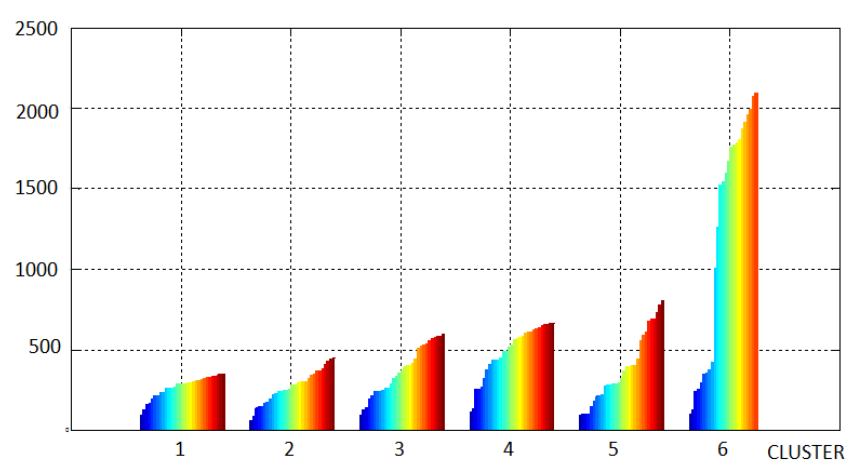

b) Modified K-means algorithm

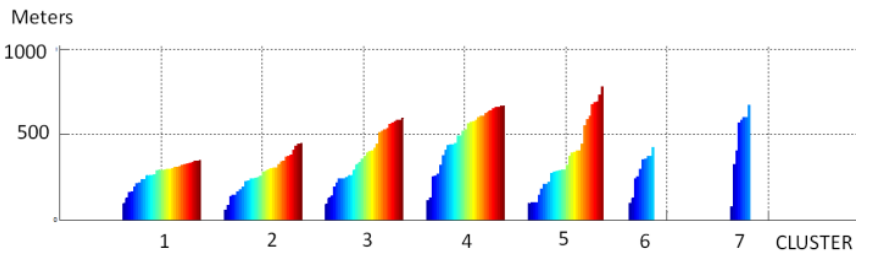

c)Clustering resulting from proposed CMPMT

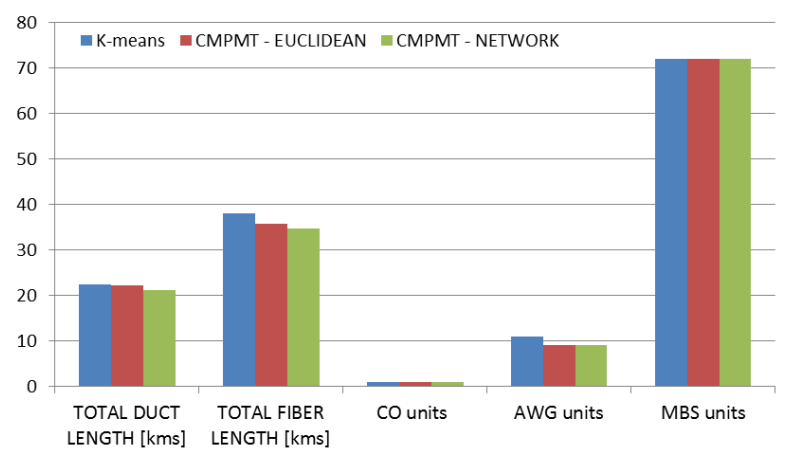

d) Clustering comparison

Figure 3 Clustering algorithm comparison: The proposed CMPMT algorithm (a) may result in higher number of clusters ((c) has one cluster more than (b) but it reduces the required fiber).

\section{PROPOSED END TO END (E2E) PROTECTION SCHEMES}

This paper proposes different E2E protection schemes which can be applied to different network points e.g., RN1, RN2, MBS. However, in this paper we apply different implementations of the schemes proposed by the authors in [15] to the E2E protection of MCO-MBS connections due to their high requirements in terms of capacity and reliability as well as their high impact factor (i.e. number of affected end users).

\section{A. Disjoint Fiber Protection (DFP)}

This scheme is based on the Type A protection scheme proposed in ITU-T G.983.1, but applied to FF and DF of each MBS. As it is shown in Figure 4 (a), the scheme needs disjoint $\mathrm{FF}$ as well as DF to each MBS. This scheme requires the following additional equipment (i) one Optical Switch (OS) at each PON LT, (ii) one OS at each MBS, and (iii) two couplers and two AWGs at each RN1.

\section{B. Ring Feeder Fiber Protection (RFFP)}

This scheme proposes connecting all the AWG through a duct ring, instead of dedicating a disjoint FF so that protection is achieved more easily (by an increase of working duct, protection is possible by using counter wise fiber). The scheme is as DFP depicted in Figure 4 (a), but in this scheme RN1 are interconnected with a ring (i.e. working fiber will be clockwise and protection fiber anti-clockwise or vice-versa depending on the location of the RN1). The working FF is the shortest fiber path, whereas the protection is the longer one. The ring is computed using the Travelling Salesman Problem (TSP) [6]. The TSP available at ArcGIS is based on a tabu search-based algorithm to find the best sequence of visiting the stops by preserving the first and last node (either AWG or MCO). From AWG to the MBS, a disjoint DF as in the DFP scheme is proposed. This scheme requires the same components and it has the same Reliability Block Diagram (RBD) as the DFP but with different FF lengths. 


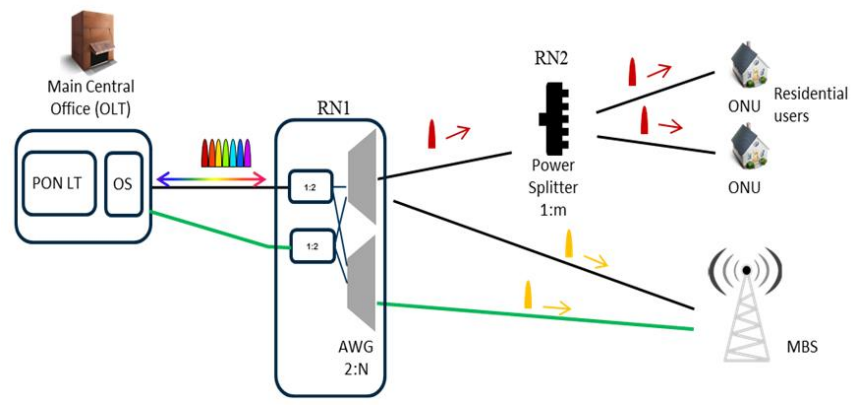

(a)

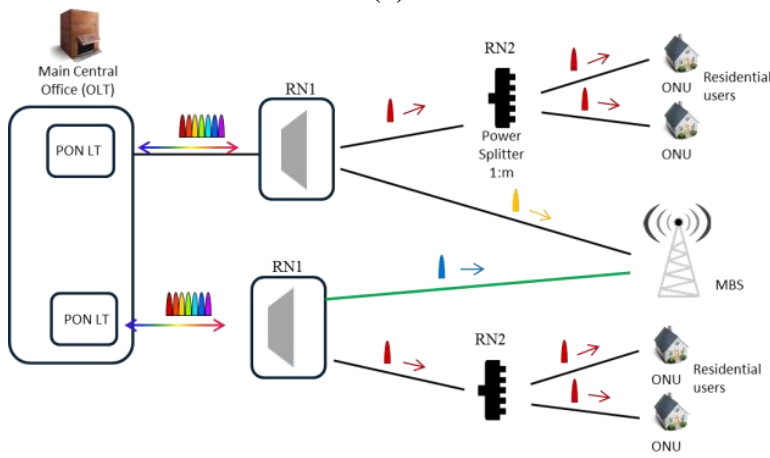

(b)

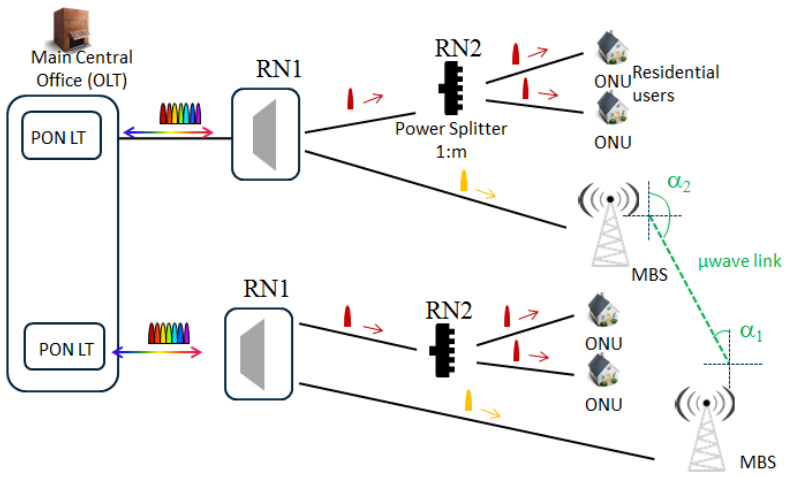

(c)

Figure 4 (a) Disjoint Fiber Protection (DFP) (b) Inter MBS Protection (IMBSP) and (b) $\mu$ Wave Protection $(\mu \mathrm{WP})$

\section{Inter MBS DF Protection (IMBSP)}

This protection scheme offers protection to MBS using a disjoint DF from the protected MBS to the closest disjoint AWG. In this case, a disjoint AWG is the one which does not share any duct with the FF and DF of the protected MBS as depicted in in Figure 4 (b). This scheme requires one OS and one filter at each MBS, since the wavelengths use for working and protection may not be the same.

\section{Ring Inter MBS Protection (RIMBSP)}

This scheme proposes connecting all the AWG through a duct ring as proposed in RFFP, so FF protection is ensured by the ring (i.e. the shorter path in any of the clockwise or anticlockwise direction is taken as working path and the other direction is allocated for protection path). For DF protection a disjoint protection path to the nearest disjoint $\mathrm{AWG}$ is required. It is expected that by proposing this scenario the solution space/probability of finding the nearer disjoint AWG to MBS is increased, thus the total DF required for protection paths will decrease. Similarly to IMBSP, RIMBSP requires: (i) one OS at each PON LT (ii) one coupler at each RN1 (iii) one OS and one filter at each MBS.

\section{E. Microwave MBS Protection ( $\mu$ WP)}

This protection scheme proposes wireless solutions to offer protection links for feeder and/or distribution segments depicted in Figure 4 (c). It offers protection to MBS based on a microwave link between two disjoint MBSs subject to two constraints (1) MBS are disjointly connected to the MCO (fiber sharing restriction) (2) MBS have a Clear Line of Sight (CLOS) ( $\mu$ Wave communication restriction). This scheme requires one microwave link for each pair of MBS and hence, the capacity of the $\mu$ Wave link should support the capacity of one MBS. Hence, for $N \mathrm{MBS}, N / 2$ microwave links are required, since the microwave link is considered to be bidirectional.

\section{ASSESSMENT METHODOLOGY}

This section extends the assessment methodology proposed in [15], which will be applied to compare the different proposed protection scenarios.

This section introduces the terms and parameters used in this assessment.

- $\quad$ FF: Feeder Fiber required for working paths.

- DF: Distribution Fiber required for working paths.

- FF': Feeder Fiber required for protection paths.

- DF': Distribution Fiber required for protection paths.

- $\quad \Delta \mathbf{W}$ : Increase of working feeder fiber with respect to the unprotected solution. The schemes based on FF rings have a non-zero $\Delta \mathrm{W}$ value.

- DUCT: Duct required by unprotected scenario.

- DUCT': Duct required by selected protection scheme.

- Average Protection Fiber Required used for availability calculations: $\frac{F F^{\prime}}{\# R N 1}+\frac{D F^{\prime}}{\# M B S}$

- Average Protection Fiber Required used for cost calculations: $\frac{F F^{\prime}+\triangle W+D F \prime}{\# M B S}$

The total protection fiber required is the heart and soul to determine the efficiency of any protection scheme. More fiber requirement means more cost and less availability which is not desired by any operator/ customer. For calculation of working paths we have used Dijkstra's shortest path algorithm with duct sharing to reduce the initial investment; for protection paths we have used Dijkstra's shortest path without duct sharing to minimize the length of required fiber. The network analyst extension of ArcGIS has been used and summarized results are shown in Table 2. It is highlighted that $\mu \mathrm{WP}$ does not require extra fiber, since it relies only on the microwave link between MBS (i.e. extra equipment cost).

\section{A. Assessment criteria}

Different parameters have been considered:

- Component Cost per MBS: This parameter considers the cost of the additional components required for the MBS protection (e.g., splitter, AWG, reach extender if required). 
The cost values are given in Cost Units (CU) which are normalized to the cost of a GPON ONU, i.e., around $50 €$.

- Power consumption per MBS: since power of access networks has been shown to be dominant consuming segment, the power of the components required for protection should be evaluated. AWGs and power splitters are passive components.

- Connection availability is defined as the probability of the connection being operational at any point of time. In this study, it corresponds to the connection between OLT and the MBS. The connection availability can be computed using the availability expression of the associated Reliability Block Diagram [13].

- Indirect improvement in connection availability of residential users: Although the objective of these protection schemes is to increase the connection availability between MCO-MBS, some schemes also increase the connection availability between MCO-Residential users.

- Failure Impact Factor (FIF) is defined as the number of affected users/connections when a particular failure occurs [10]. The FIF of an unprotected component is computed as the product of its unavailability and its FPR. The FIF of any connection can be computed as the sum of the FIF of each involved unprotected component.

- $\quad$ Protection fiber length required per MBS: How much fiber should be installed in order to protect the end points. In general, the longer the fiber, the more expensive is the solution.

- $\Delta W$ (Additional Fiber Requirement for Working Paths): The protection schemes considering an FF ring require longer working FF than the other schemes which consider working FF as shortest path between MCO and RN1. This difference, denoted as $\Delta \mathrm{W}$, is important when aiming at comparing the investment required for the different schemes.

\section{B. Comparative overall performance}

The comparison is based on two techniques:

- Spider Net Diagram Comparison: The first step is to discretize the values of each parameter. The diagram will have as many axes as parameters. The parameters for one particular scenario/area will be plotted and compared: the smaller the diagram, the better is the scheme for that area.

- Pondered assessment: Each operator may prioritize some parameters and hence, the importance of those should have higher weight than the others. E.g., an operator could prioritize the required investments and hence, select as most important parameters the Component cost, Protection fiber length/MBS and $\Delta \mathrm{W}$. Another operator could prioritize the customer satisfaction and hence, the parameters with higher weights would be connection availability and indirect improvement in connection availability of residential users.

\section{CASE STUDY}

Let us perform the comparative assessment of the proposed protection schemes into three areas with different building and MBS densities:
- Dense Urban (DU): 2863 buildings and $72 \mathrm{MBS}$ in a $3 \mathrm{~km}^{2}$ area (Berlin).

- Urban (U): 2462 buildings and $70 \mathrm{MBS}$ in a $12 \mathrm{~km}^{2}$ area (Helfenberg).

- Rural (R): 3103 buildings and 64 MBS in a $45 \mathrm{~km}^{2}$ area (Miesbach).

In this case, a two-stage optical access architecture has been considered with AWGs of 1:40 wavelengths at the first remote node and PS of 1:32 splitting ratio at the second remote node. The maximum reach is $17 \mathrm{~km}$ for residential users and $43 \mathrm{~km}$ for MBS (D4.2.1 of the OASE project). The port utilization, i.e., the maximum number/ upper ceiling of ports which are allowed to be used, is set to $80 \%$. The remaining $20 \%$ of the ports are left for protection or future use. The data considered in this study has been obtained from [12].

The values obtained for each of the proposed parameters have been summarized in Table 1 :

- Component Cost per MBS: DFP and RFFP have the same component costs since they only differ on the fiber layout. RIMBSP is having less cost than DFP and RFFP due to the less required equipment. IMBSP requires the minimum additional equipment, so it is the most economical. $\mu \mathrm{WP}$ is the most expensive one, but it gives more flexibility and offers quick installation.

- Power consumption per MBS: Since the power consumption of optical switches are much lower than the power consumed by filters, DFP and RFFP consume much less power than the other schemes. The $\mu \mathrm{WP}$ has the highest power consumption despite the two modes of operation of the $\mu$ Wave link (sleep and active).

- Connection availability: The components and the fiber availability reference values have been taken from [3] and also explained in [13]. IMBSP, RIMBSP and $\mu \mathrm{WP}$ schemes have higher connection availability due to the PON LT protection at the OLT. $\mu \mathrm{WP}$ offers even higher availability due to the duplication of all the components (incl. ONU). Despite of the different fiber lengths of DFP and RFFP, they show comparable connection availability because the fiber is protected, and hence, its length has very little impact on connection availability.

- Indirect improvement in connection availability of residential users (presented as a percentage with respect the unprotected case): DFP, RFFP and RIMBSP increase the availability of residential users, because the protected FF is common to MBS as well as to residential users. However, IMBSP and $\mu$ wave do not improve residential user availability. The degree of improvement depends on the area as the length of FF and FF' is different. In $\mathrm{U}$ and $\mathrm{R}$ areas the availability improvement is higher due to the longer impact of protecting FF (they are longer than in DU areas). The resultant the availability of residential users (is almost four-nines compared with the three-nines unprotected availability).

- Failure Impact Factor (FIF): The FIF of $\mu \mathrm{WP}$ scheme is zero as all components are protected. FIF of IMBSP and RIMBSP is very low as PON LT is protected in these schemes. DFP and RFFP are having the highest FIF because they have a larger set of unprotected components. As both schemes are using the same architectural scheme 
the FIF values are the same. Compared to the unprotected scenario (FIF value $=0.0265186717$ ), DFP and RFFP schemes have decreased FIF by almost $50 \%$, IMBSP and RIMBSP has decreased the FIF by almost 50,000 times.

- Protection fiber length required per MBS: It can be observed that DFP is the most economical solution in DU and $U$ areas. However, when the area becomes too sparse like $\mathrm{R}$ area, IMBSP turns to be the most economical solution.

- $\Delta W$ : The schemes with ring protection show an increase of fiber of almost $40-50 \%$ in DU and $U$ areas and $300 \%$ in R areas.

Table 1 Assessment parameters for each protection scheme and area type

\begin{tabular}{|c|c|c|c|c|c|c|c|}
\hline & $\begin{array}{c}\text { Component } \\
\text { cost/MBS } \\
\text { [CU] }\end{array}$ & $\begin{array}{c}\text { Power } \\
\text { consumption/ } \\
\text { MBS [kWh] }\end{array}$ & $\begin{array}{l}\text { Connection } \\
\text { availability }\end{array}$ & $\begin{array}{c}\text { Decrease } \\
\text { Residential } \\
\text { users } \\
\text { unavailability }\end{array}$ & FIF & $\begin{array}{l}\text { Prot. } \\
\text { Fiber } \\
{[\mathrm{km}]}\end{array}$ & $\begin{array}{c}\Delta \mathrm{W} \\
{[\mathrm{km}]}\end{array}$ \\
\hline \multicolumn{8}{|c|}{ DU } \\
\hline DFP & 5,19 & 0,001 & 0,999912809 & $6,3 \%$ & 0,017735 & 0,87 & 0 \\
\hline RFFP & 5,19 & 0,001 & 0,999912808 & $6,3 \%$ & 0,017735 & 1,16 & 3,109 \\
\hline IMBSP & 3,5 & 12,265 & 0,999948996 & $0,0 \%$ & 0,000051 & 1,13 & 0 \\
\hline RIMBSP & 3,79 & 12,625 & 0,999948995 & $5,6 \%$ & 0,000051 & 1,33 & 3,109 \\
\hline$\mu \mathrm{WP}$ & 75 & 17,522 & 0,999999987 & $0,0 \%$ & 0,000033 & 0 & 0 \\
\hline \multicolumn{8}{|c|}{$\mathbf{U}$} \\
\hline DFP & 4,81 & 0,001 & 0,999912809 & $17,0 \%$ & 0,017849 & 2,42 & 0 \\
\hline RFFP & 4,81 & 0,001 & 0,999912808 & $17,0 \%$ & 0,017849 & 2,85 & 4,962 \\
\hline IMBSP & 3,5 & 12,265 & 0,999948996 & $0,0 \%$ & 0,000051 & 2,8 & 0 \\
\hline RIMBSP & 3,76 & 12,625 & 0,999948995 & $16,4 \%$ & 0,000051 & 2,9 & 4,962 \\
\hline$\mu \mathrm{WP}$ & 75 & 17,522 & 0,999999987 & $0,0 \%$ & 0,000033 & 0 & 0 \\
\hline \multicolumn{8}{|c|}{$\mathbf{R}$} \\
\hline DFP & 5,08 & 0,001 & 0,999912809 & $14,3 \%$ & 0,022314 & 5,74 & 0 \\
\hline RFFP & 5,08 & 0,001 & 0,999912808 & $14,2 \%$ & 0,022314 & 7,95 & 31,078 \\
\hline IMBSP & 3,5 & 12,265 & 0,999948996 & $0,0 \%$ & 0,000051 & 4,51 & 0 \\
\hline RIMBSP & 3,78 & 12,625 & 0,999948995 & $13,7 \%$ & 0,000051 & 7,28 & 31,078 \\
\hline$\mu \mathrm{WP}$ & 75 & 17,522 & 0,999999987 & $0,0 \%$ & 0,000033 & 0 & 0 \\
\hline
\end{tabular}

The assessment of the parameters in Table 1 is not straightforward and hence, we apply the two techniques proposed in Section VI.B.

- Spider Net Diagram Comparison: In our study we have discretized the values of Table 1 to four (from 1-best to 4-worst) as proposed in [12]. Based on the values, the spider net diagrams can be generated (depicted in Figure 5 (a) and (b) for DU and $U$ areas respectively). The advantage of this technique is that this graphical representation helps comparing schemes: in this case, RIMBSP is clearly better than RFFP (except for power consumption) since it covers a smaller area. This diagram also helps understanding how each protection scheme is affected by the area, (e.g., $\mu \mathrm{WP}$ does not depend significantly on the area type, whereas RFFP does.)

Looking at the diagrams the first conclusion is that there is no clear winner and hence, a compromise should be considered. We can also deduce that the connection availability and FIF do not vary significantly on the area since they are driven by the unprotected components, which do not include the fiber. The $\mu \mathrm{WP}$ scheme shows extreme values (either best or worst) and does not depend on the area type.

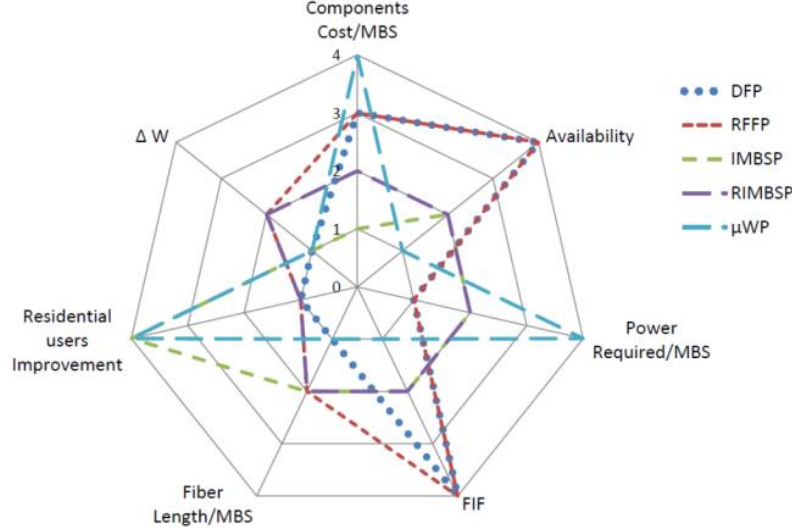

a) Spider Net Diagram for Dense Urban area

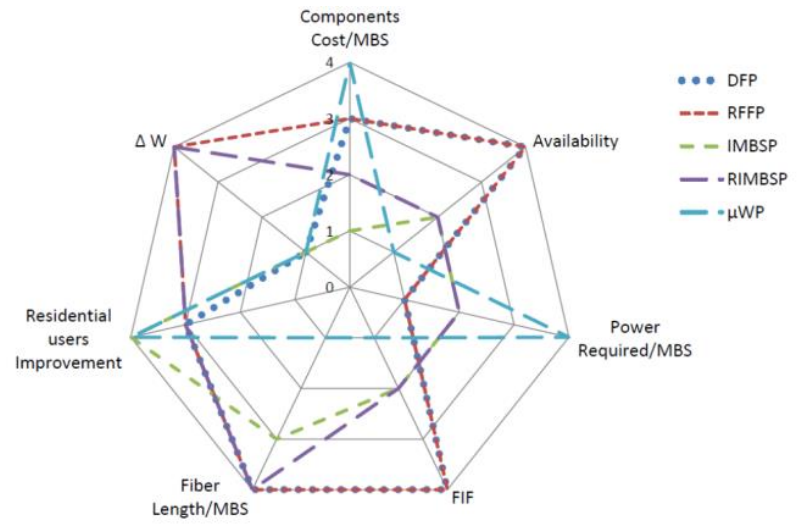

b) Spider Net Diagram for Rural area

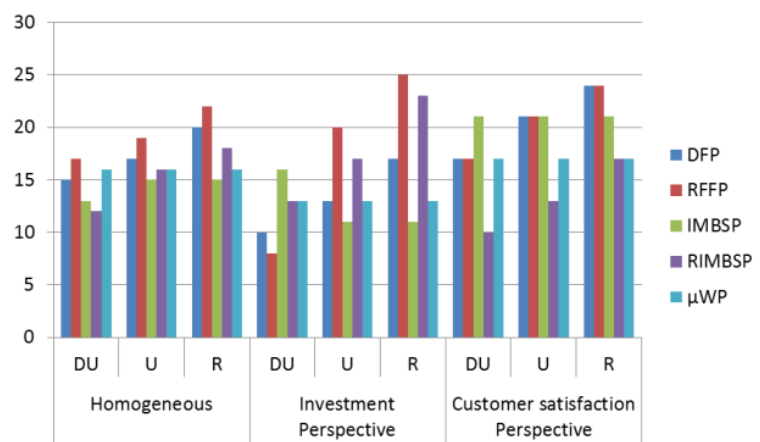

c)Pondered comparative diagram where the y-axis is the sum of all discretized parameters of each scenario (i.e. 8 is best and 25 is the worst

Figure 5 Assessment Diagrams

- Pondered assessment: Since there is no best solution, the priorities of each operator should be taken into account when evaluating the protection schemes. Fig. 5 (c) compares the schemes for the different interests (e.g., investment perspective prioritizes component cost, protection fiber length and $\Delta \mathrm{W}$ with respect the other parameters; whereas the Customer satisfaction perspective prioritizes connection availability to MBS and residential users). This ponderation should be adjusted based on the interests of each operator. In this case, an operator concerned with the investment, would choose RFFP in DU areas and IMBSP for $\mathrm{U}$ and $\mathrm{R}$ areas. An operator 
prioritizing the customer satisfaction would choose RIMBSP for any type of area.

\section{VIII.DISCUSSION AND CONCLUSION}

A Converged Access Network Planning and Dimensioning Tool has been described. This tool incorporates a new clustering algorithm which aims at reducing the required infrastructure. The proposed tool has been used to compare different proposed protection architectures for converged access networks in different types of areas. The best protection scheme depends on the clear and concise requirements of the operator and the deployment area. Comparative and consolidated performance analysis of each protection scheme has been carried out with even and uneven weights distribution to select the best protection scheme in a particular scenario/ area. It has been shown that with even weights distribution RIMBSP is the best protection scheme in DU area and IMBSP is the best protection scheme in urban and rural areas. Besides, these weights can be adjusted by service/ network provider to meet any specific goal/ requirement. It is also shown that by changing these weights the results significantly vary; thereby the best solutions in the three types of areas depend on the prioritized criteria by the operator.

\section{REFERENCES}

[1] M. Forzati et al. "Next-generation optical access seamless evolution: Concluding results of the European FP7 Project OASE," Journal of Optical Communications and Networking, vol. 7, no. 2, pp. 109-123, 2015

[2] D. Gardan et al., "Techno-economics of advanced optical subscriber networks," Global Telecommunications Conference and Exhibition 'Communications Technology for the 1990s and Beyond' (GLOBECOM), 1989. IEEE, pp. 1335-1339 vol.3, November 1989

[3] K. Casier, "Techno-economic evaluation of a next generation access network deployment in a competitive setting," $\mathrm{PhD}$ degree at the Faculty of Engineering of the Ghent University, October 2009

[4] D. Maniadakis and D. Varoutas, "Incorporating Gabriel graph model for FTTx dimensioning," Photonic Network Communications, vol. 29, no. 2, pp. 214-226, 2015.

[5] B. Olsen et al., "RACE 2087/TITAN: Tool for Introduction scenarios and Techno-economic studies for the Access Network," Proc. RACE open workshop on broadband access, pp. 7-8, 1993.

[6] A. Mitcsenkov et al. "Geometric versus geographic models for the estimation of an FTTH deployment," Telecommunication Systems, vol 54, no. 2, pp. 113-127, 2013

[7] O. Kipouridis et al. "Street-aware infrastructure planning tool for Next Generation Optical Access networks," Optical Network Design and Modeling (ONDM), 2012 16th International Conference on, pp. 1-6, April 2012

[8] C. Lange et al., "Effects of network node consolidation in optical access and aggregation networks on costs and power consumption," OPTO, pp. 76 210F-76 210F, 2010.

[9] M. Mahloo, "Transport Solutions for Future Broadband Access Networks," Ph.D. dissertation, KTH, Stockholm, Sweden, 2015

[10] M. Mahloo et al., "Toward reliable hybrid WDM/TDM passive optical networks," Communications Magazine, IEEE, vol. 52, no. 2, pp. S14-S23, February 2014

[11] R. H. Nielsen et al., "On the potential of using the cable trench problem in planning of ICT access networks," 50th International Symposium ELMAR, vol. 2, pp. 585-588, 2008

[12] A. Shahid et al. "Comparative Analysis of Protection Schemes for Fixed Mobile Converged Access Networks based on Hybrid PON," 12th International Conference of Telecommunication, Media and Internet Techno-Economics (CTTE), November 2015
[13] A. Shahid, "Enhanced dimensioning and comparative analysis of different protection schemes for Hybrid PON Converged Access Networks (HPCAN)," Master's thesis, TRLKN-2015-02, Technical University Munich (TUM), Germany, 2015

[14] B. Kanj, C. Mas Machuca, K. Grobe, "Comparison of Wavelength-Routed and Wavelength-Split WDM-PON in Mobile x-Haul in dense urban areas", ITG-Fachtagung Photonische Netze 2016, Leipzig, Germany, May 2016

[15] C. Mas Machuca, L. Wosinska, J. Chen, "Assessment methodology of protection schemes for next generation optical access networks", Optical Fiber Technology, pp. 82-93, December 2015 